\title{
Genome-wide association and targeted analysis of copy number variants with psoriatic arthritis in German patients
}

\author{
Steffen Uebe ${ }^{1}$, Maria Ehrlicher ${ }^{1}$, Arif Bülent Ekici', Frank Behrens², Beate Böhm², Georg Homuth³,
} Claudia Schurmann ${ }^{3}$, Uwe Völker $^{3}$, Michael Jünger $^{4}$, Matthias Nauck $^{5}$, Henry Völzke ${ }^{6}$, Heiko Traupe ${ }^{7}$, Michael Krawczak ${ }^{8}$, Harald Burkhardt ${ }^{2}$, André Reis $^{1}$ and Ulrike Hüffmeier ${ }^{1 *}$ (D)

\begin{abstract}
Background: Psoriatic Arthritis (PsA) is a chronic inflammatory disease of the joints. PsA is etiologically complex, and 11 susceptibility loci have been identified so far. Most of these overlap with loci associated with psoriasis vulgaris (PsV), the most common psoriatic skin manifestation which is also frequently seen in PsA patients. In addition, two copy number variants (CNVs) are associated with PSV, one of which, located within the LCE3 gene cluster, is also associated with PsA. Finally, an intergenic deletion has been reported as a PsA-specific CNV.

Methods: We performed a genome-wide association study (GWAS) of CNVs in PsA and assessed the contribution to disease risk by CNVs at known psoriasis susceptibility loci.

Results: After stringent quality assessment and validation of CNVs of the GWAS with an alternative quantitative method, two significantly associated CNVs remained, one near UXS1, the other one at the TRB locus. However, MLPA analysis did not confirm the CN state in $~ 1 / 3$ of individuals, and an analysis of an independent case-control-study failed to confirm the initial associations. Furthermore, detailed PCR-based analysis of the sequence at TRB revealed the existence of a more complex genomic sequence most accurately represented by freeze hg18 which accordingly failed to confirm the hg19 sequence.

Only rare CNVs were detected at psoriasis susceptibility loci. At three of 12 susceptibility loci with CNVs (CSMD1, IL12B, RYR2), CN variability was confirmed independently by MLPA. Overall, the rate of CNV confirmation by MLPA was strongly dependent upon CNV type, CNV size and the number of array markers involved in a CNV.

Conclusion: Although we identified PsA associations at several loci and confirmed that the common CNVs at these sites were real, $\sim 1 / 3$ of the common CNV states could not be reproduced. Furthermore, replication analysis failed to confirm the original association. Furthermore, SNP array-based analyses of CNVs were found to be more reliable for deletions than duplications, independent of the respective CNV allele frequency. CNVs are thus good candidate disease variants, while the methods to detect them should be applied cautiously and reproduced by an independent method.
\end{abstract}

Keywords: Copy number variant, Psoriatic arthritis, SNP arrays, Genome-wide association study, TRB, Psoriasis susceptibility loci

\footnotetext{
*Correspondence: Ulrike.Hueffmeier@uk-erlangen.de

${ }^{1}$ Institute of Human Genetics, Friedrich-Alexander-Universität

Erlangen-Nürnberg (FAU), Schwabachanlage 10, 91054 Erlangen, Germany

Full list of author information is available at the end of the article
} 


\section{Background}

Psoriatic arthritis (PsA) is a common, chronic, inflammatory arthritis that is mainly observed in combination with the most common skin manifestation of psoriasis, namely psoriasis vulgaris (PsV). PsA is classified as a spondyloarthropathy and is usually sero-negative for autoantibodies. Inflammation typically manifests at peripheral large and/ or small joints, the spine, and/ or entheses.

PsA is a complex disease, because it is known to be caused by a combination of different genetic and environmental risk factors. Interestingly, most recent heritability estimates suggest a 2- to 5-fold stronger genetic component in PsA than in PsV. Thus, the relative risk of first-degree family members $\left(\lambda_{1}\right)$ in PsA equals 30 to 39 $[1,2]$ compared to $7-19$ in $\operatorname{PsV}[1,3]$. However, the number of identified genetic risk factors is much higher for PsV than for PsA because of the larger samples analyzed to date. Genome-wide association studies (GWAS) or more targeted analyses of immunologically relevant genes have accelerated the identification of susceptibility factors and $44 \mathrm{PsV}$ susceptibility loci have been confirmed at a genome-wide level of significance so far [4-12]. The majority of genetic susceptibility factors for PsA have been shown to overlap with those of PsV [9, 13-16]. This notwithstanding, a non-coding variant at $5 q 31$ overlapping with juvenile idiopathic arthritis, Crohn's disease and asthma [14] and a missense variant in PTPN22 [15] as well as the HLA-B27 locus may be more joint-specific risk factors.

Similar to other complex diseases, GWAS for PsV and PsA were focused upon single nucleotide polymorphisms (SNPs). Copy number variants (CNVs), however, are also known to contribute substantially to the diversity of the human genome $[17,18]$ and may thus be involved in the pathogenesis of both monogenic and common complex diseases as well. For PsV, an increased copy number at the highly polymorphic $ß$ defensin $(D E F B)$ gene cluster at 8p23.1 has been shown to increase disease susceptibility $[19,20]$. In addition, a frequent $32 \mathrm{~kb}$ deletion within the late cornified envelope 3 gene cluster ( $L C E 3 C \_3 B$-del) was found to be a risk factor for both PsV and PsA [5, 21-24]. More recently an intergenic deletion has been shown to be associated with PsA, but not with PsV [25].

In view of the known associations between CNVs and psoriasis and to improve our understanding of the genetic basis of PsA, we performed a GWAS of CNVs for PsA, using previously published data from our own group of PsA patients [16].

\section{Methods}

\section{Study groups and data}

To investigate the association between PsA and CNVs at a genome-wide level, we used SNP array-derived data
(Affymetrix Genome-Wide Human SNP Array 6.0) of 572 German patients with PsA [16] and of 4081 healthy individuals from a population-based control cohort (free of psoriasis) (Study of Health in Pomerania; SHIP) [26]. As described elsewhere [27], we excluded individuals with excessive numbers of CNVs, leaving a discovery cohort of 478 patients and 3798 controls. A primary component analysis based on SNP genotypes of 478 PsA patients and 3798 SHIP individuals was performed using smartpca from the EIGENSTRAT software suite [28]. We did see evidence for marginal stratification within the SHIP data set, but the vast majority of SHIP samples clustered with PsA cases in the first 6 eigenvectors, indicating no major influence of population stratification effects.

DNA samples from independent groups of PsA patients $(n=251)$ and healthy blood donors $(n=451)$, both of which represented subgroups of previously described cohorts [29], were used for the replication of the disease association of a deletion near UXS1. For the replication of the disease association of a $\mathrm{CNV}$ at $T R B$, we used DNA from 782 PsA patients, including all individuals of our discovery study, and 897 independent control individuals [30]. In order to avoid confusion, we henceforth use the term "replication" for a study that aims to confirm a disease association, whereas "validation" refers to the technical confirmation of a CNV by MLPA (see below).

\section{CNV genotyping and procedure of quality assessment in the GWAS}

SNP array-based CNV genotyping analysis and quality assessment of CNVs were done as described in detail elsewhere [27]. Briefly, we used the CNV calling algorithm Birdseye to analyse the genome-wide data [31] and considered CNVs covered by $\geq 5$ array-markers spanning $\geq 5000$ base pairs (bp), thereby identifying 26 CNVs. Within those, we identified batch effects (differences in CNV frequencies between different groups of conjointly processed microarrays) as a major confounder and corrected for it with a linear mixed model analysis. In this latter analysis, association to $12 \mathrm{CNVs}$ was not confirmed. Within the $26 \mathrm{CNVs}$, we observed further problematic issues that partly overlapped in single CNVs: association to CNVs' boundary markers only $(n=4)$, no evidence for differences in CNV frequency when compared to one or both of two smaller control groups $(n=11)$, location in regions of high and/ or extended sequence homology $(n=18)$. A correlation analysis provided evidence that certain characteristics of the CNVs as well as problematic issues were not independent from each other. Problematic issues were more common in the $12 \mathrm{CNVs}$ with no confirmation of association in the linear mixed model. 
Mainly within the $14 \mathrm{CNVs}$ that passed the linear mixed model and provided less/ no evidence for the further problematic issues, we selected $11 \mathrm{CNVs}$ for validation by an independent quantitative method, namely multiplex ligation-dependent probe amplification (MLPA). For $8 \mathrm{CNVs}$, probe pairs could be self-designed as described [27, 32], and MLPA was performed as recommended by the manufacturer (MRC Holland, Amsterdam, The Netherlands) and as described in more detail elsewhere [27, 32]; in case of three CNVs, a probe design was impossible. Figure 1 exemplifies the analysis of relevant CNVs. At least five control individuals with two gene copies at all reference loci (one gene copy in the case of the TRB deletion) were included in each experiment as internal controls. Every target $\mathrm{CNV}$ was covered by at least one pair of CNV-specific MLPA probes that captured the most common $\mathrm{CNV}$ at the respective locus. Individuals with one of the three $\mathrm{CN}$ states (wild-type (WT)/ WT; WT/ CNV, CNV/ CNV) were re-analysed by MLPA. Since no DNA was available for the SHIP controls, however, their CNV genotypes could not be confirmed by MLPA.

CNVs at two loci passed both quality assessment and validation with MLPA. These CNVs either neighboured
UXS1 or were located at the TRB locus (size and position in different genomic annotations as indicated in Additional file 1: Table S1).

\section{Molecular analyses at the TRB locus}

Since most of the array markers spanning the CNV at the TRB gene were only present in the hg18 human genome assembly, but not in hg19, we analysed this locus both in silico and experimentally. To this end, we aligned hg18 and hg19 using the Mauve software (http:// darlinglab.org/mauve/), and performed additional laboratory experiments to infer which of the two assemblies was more reliable at TRB. We established four different PCRs with oligonucleotides specific to the sequence in hg18 and two PCRs specific to hg19 (Fig. 2). PCR and Sanger sequencing following standard protocols [29] were performed in Eppendorf Mastercyclers (Eppendorf, Hamburg, Germany). Purification of PCR products (Agencourt AMPure kit) and sequencing reactions (CleanSEQ) were performed using the BeckmanCoulter Biomek Nxp robotic system (Beckman-Coulter, Krefeld, Germany). MLPA identified one of the three possible $\mathrm{CN}$ states $(0,1,2$ copies) at the TRB locus in 135 of individuals. For 10 of these individuals (5 with 0

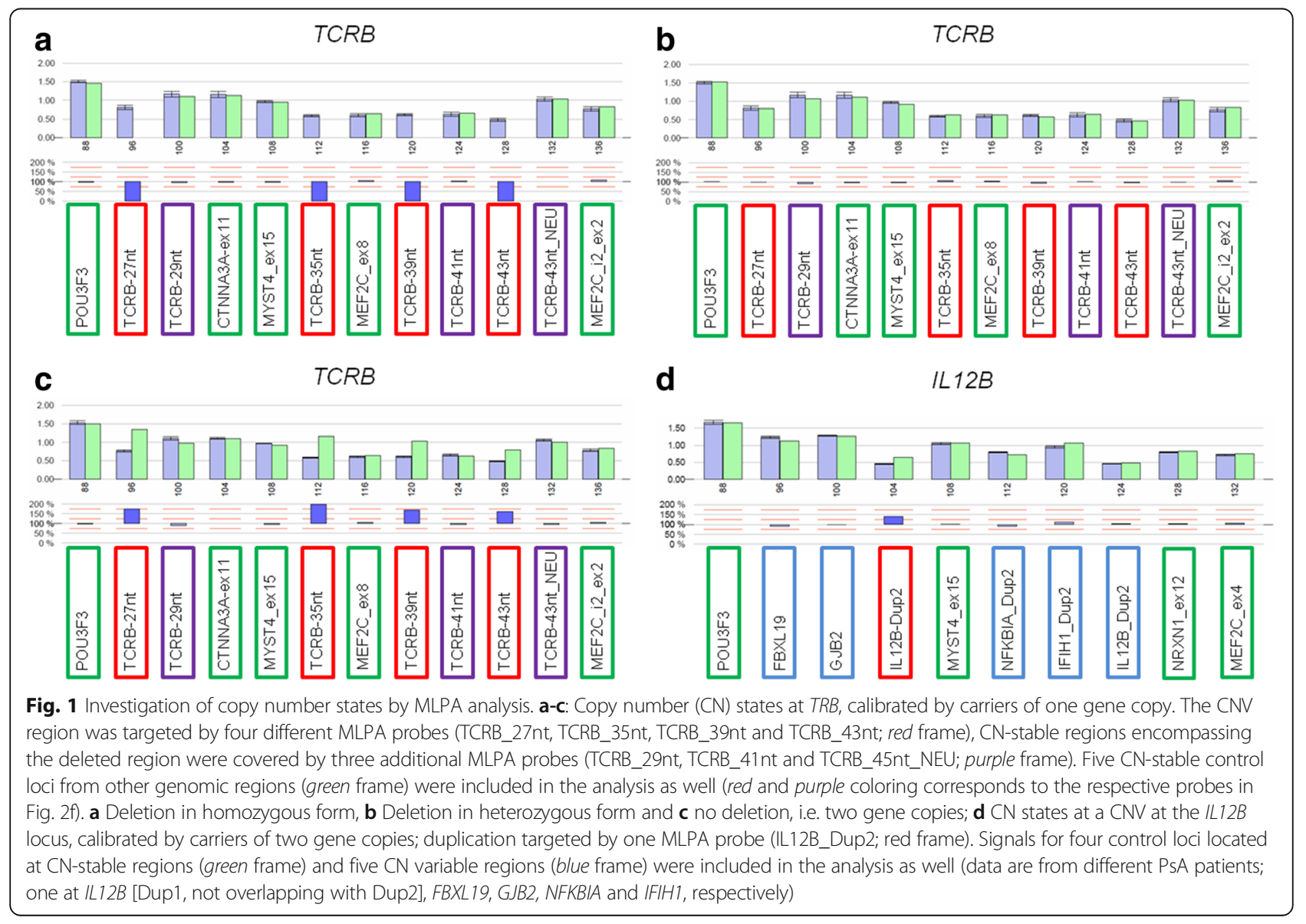




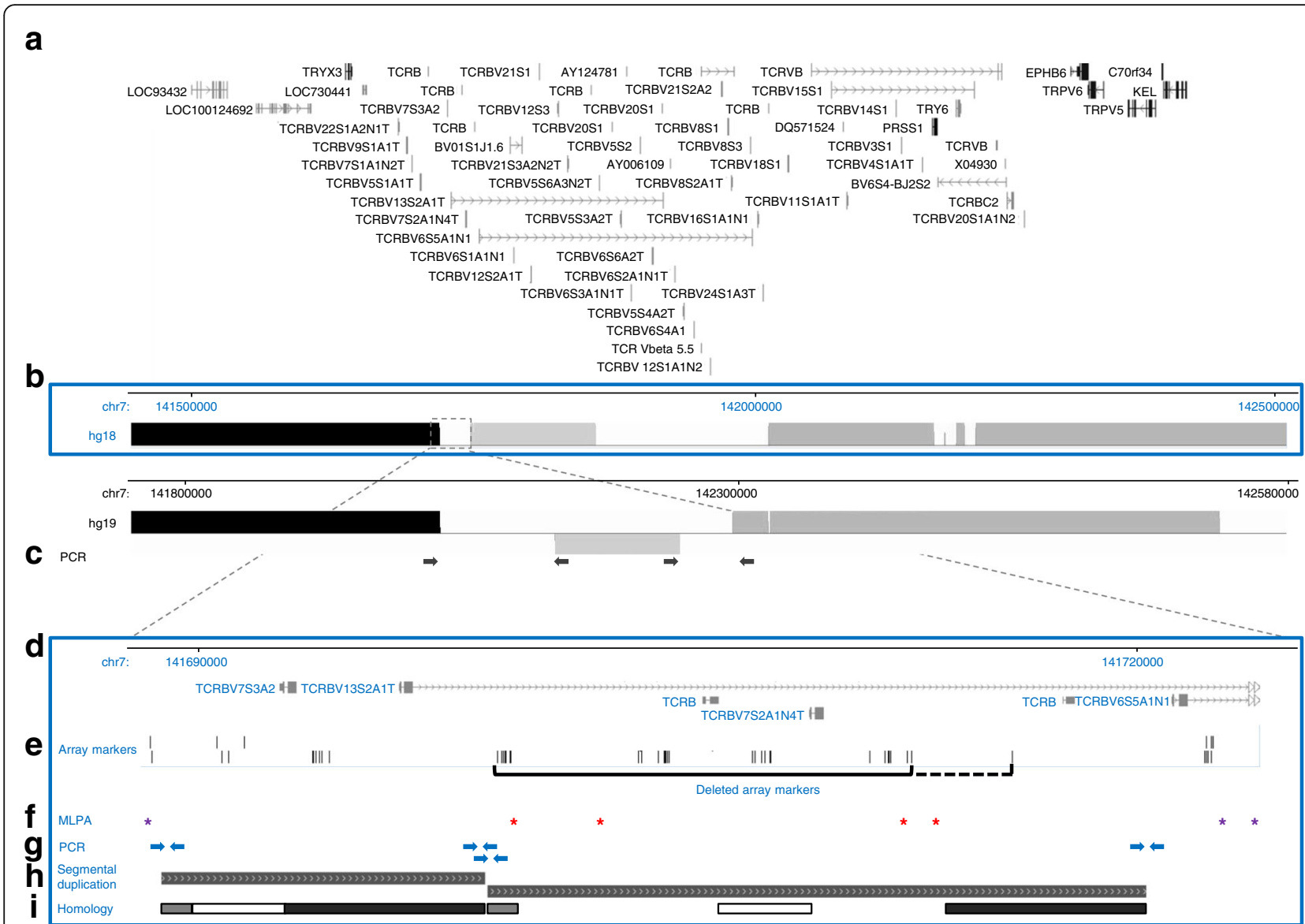

Fig. 2 Genomic annotation of the TRB gene cluster on chromosome 7q34. a Annotated UCSC genes in NCBI36/hg18 (genome-euro.ucsc.edu); b Sequence alignments of genome freezes NCBI36/hg18 and GRCh37/hg19 with chromosomal positions, similar or identical sequences (grey and black bars, respectively), unique/ unaligned sequences (white), sequences identified to be inverted in freeze hg19 are given below the baseline; c PCR products specific for inversion GRCh37/hg19 (arrows); d-i Magnification (dotted box/ lines) of genome freeze NCBI36/hg18; d Genomic position of frequent TRB deletion, chromosomal position and UCSC genes; e Positions of array-markers: upper row = SNP markers; lower row = CN markers; solid black line = most common, minimal size of deletion (13,136 bp or 28 markers). The dashed line indicates one additional array-marker that is commonly deleted as well (deletion of 16,338 bp or 29 markers); $\mathbf{f}$ Positions of pairs of MLPA probes (asterisks) encompassing the TRB locus. Probes marked in purple encompass the CNV, probes marked in red are located within the deletion (see Fig. 1a-c); $\mathbf{g}$ Arrows indicate PCR primers for amplicons specific to NCBI36/hg18 (arrows); h Segmental duplication of 90-98\% similarity (grey patterned bars); i Homologous sequences in the first and second part of the segmental duplication (indicated by bar of same color)

copies, 3 with 1 copy, and 2 with 2 copies), however, PCR failed to yield products specific for genome freeze hg19 at all, whereas DNA of 19 individuals (5 with no copies, 11 with 1 copy and 3 with 2 copies) yielded PCR products specific to hg18. All sequences were analysed using SeqMan software (DNASTAR, Madison, USA) and were aligned to the respective reference sequences (NCBI36/hg18 and GRCh37/hg19).

\section{Analysis of CNVs at psoriasis susceptibility loci}

In addition to a GWAS, we analysed CNVs at 30 psoriasis disease loci of at least borderline genome-wide significance published until November 2010 [4-11, 13, 16]. First, we investigated the degree of linkage disequilibrium (LD) in our GWAS data [16] between the most significantly disease-associated SNP at each locus and the surrounding SNPs, using HaploView 4.2 (https://www.broadinstitute.org/haploview/haploview). Regions of relevant LD with the most significantly associated SNP were defined either as previously described [33] or by having an $\mathrm{r}^{2}>0.1$. In a second step, $\mathrm{CN}$ data of PsA patients were screened for CNVs overlapping these LD regions as described elsewhere [27]. In order to validate the disease association of $\mathrm{CNVs}$ in the overlapping regions, an MLPA probe was designed for each disease locus at which CNVs were identified in our PsA cohort (feasible for all but one locus). The probes were found to cover the majority of the observed CNVs (Additional file 1: Table S2). A subset of 7-14 PsA patients including carriers of the rare $\mathrm{CNV}$ were included in the validation step by MLPA. 


\section{Additional statistical analyses}

For each CNV detected in our study $(n=28$; Tables $1,2,3$, Additional file 1: Table S2), MLPA results were compared to the genotypes determined in the GWAS [27]. To this end, we defined a CNV-specific match score for each individual that was set equal to 2 , if both genotypes were found to be of the same type and copy number; a match score of 1 was assigned, if the two genotypes matched only in type (i.e. deletion or duplication), but not in copy number; otherwise, the match score was set to 0 . Next, the mean match score taken over all individuals was determined for each CNV. To assess the influence of the $\mathrm{CNV}$ type (deletion or duplication) on the degree of MLPA validation, we compared the match score distributions obtained for the two types of CNVs using a Mann-Whitney rank sum test. To assess the influence of the segment size and number of array-markers involved in a CNV, we first determined the median segment size and median marker count for each of the $28 \mathrm{CNVs}$. Then, the mean was calculated over the mean match scores of all loci with a median size or median marker count larger than the CNV-specific value (Fig. 3). Finally, Spearman's rank correlation coefficient $\rho$ was calculated as a correlation coefficient between those means and the median $\mathrm{CNV}$ size or marker count, respectively.

\section{Results}

In the GWAS, we observed an association with PsA of the CNVs near UXS1 and at the TRB locus (Tables 1 and 2). The respective copy number states at these loci were confirmed by MLPA (Fig. 1a-c).

\section{CNVs at the UXS1 locus}

One of the disease associated CNVs was intergenic, located $69.3 \mathrm{~kb}$ upstream of UXS1 on one side and $113.2 \mathrm{~kb}$ upstream of PLGLA on the other side. The UXS1 gene encodes UDP-glucuronate decarboxylase, an enzyme that catalyses the formation of UDP-xylose from UDP-glucuronate used in the biosynthesis of glucosaminoglycans. PLGLA is a pseudogene of the gene encoding plasminogen, a major dissolvent of fibrin. The UXS1 and PLGLA genes are positional candidates for PsA causality, especially $U X S 1$, although we cannot exclude that other genes in the vicinity might also be affected by the deletion. Our association findings were not replicated in an independent sample of 251 PsA patients and 451 controls (Table 1). Although we could confirm the deletion and wild-type states in 85 of 135 PsA patients genotyped with both a SNP array and MLPA, discordant $\mathrm{CN}$ states emerged for 50 of 135 individuals (37.0\%). We therefore re-analysed the SNP-array based $\mathrm{CN}$ data as described elsewhere [27] and identified smaller CNVs that had been excluded because one filter criterion for exclusion was a minimal size of $5000 \mathrm{bp}$.

When the frequent, albeit short CNVs were included in the initial association study, equal allele frequencies of the deletion were observed in PsA patients and control individuals (33.6\% vs. $32.3 \%)$.

\section{Deletion at the TRB locus}

The TRB locus encodes a $\mathrm{T}$ cell receptor, a molecule responsible for recognizing foreign antigens. Therefore, the frequent deletion detected at the TRB locus was recognized as a most interesting candidate for PsA causality. Comparison of the SNP array-based MLPA-based genotypes revealed discrepancies for 48 of 135 individuals (35.6\%). For this CNV, we could exclude that smaller frequent CNVs - that escaped due to our filter criterion of a minimal size of $5000 \mathrm{bp}$ as described in case of the CNV near UXS1 - caused a false positive association. We decided to analyse this $\mathrm{CNV}$ in more detail. Seven different pairs of MLPA probes located either within the deletion or flanking it (hg18 assembly) (Fig. 2f) were used for genotyping and similar results were obtained with both types of probes (same coloring of target probes in Fig. 1a-c and Fig. 2f).

Sequence analysis and genomic alignment revealed different annotations in human genome freezes hg18 and hg19 (Fig. 2b). Thus, SNP array-markers identified to be disease-associated in our genome-wide study were not annotated in hg19, and a segmental duplication within the region was annotated only in hg18 (Fig. 2b, h). In addition, an inversion of a $118 \mathrm{~kb}$ was only present in hg19 (Fig. 2b). To investigate the differences in genomic

Table 1 Allele counts (frequencies) of the risk/ non-risk alleles [n (\%)] of the CNV near UXS1

\begin{tabular}{|c|c|c|c|c|c|c|c|c|}
\hline $\begin{array}{l}\text { absolute chromosomal } \\
\text { position (hg18) }\end{array}$ & $\begin{array}{l}\text { relative position to } \\
\text { nearest gene(s) }\end{array}$ & Part of study & $\begin{array}{l}\mathrm{n}(\%) \text {-risk } \\
\text { allele in PsA }\end{array}$ & $\begin{array}{l}\mathrm{n}(\%)-\text { non-risk } \\
\text { allele in PsA }\end{array}$ & $\begin{array}{l}n(\%)-\text { risk } \\
\text { allele in Ctrl }\end{array}$ & $\begin{array}{l}\mathrm{n}(\%)-\text { non-risk } \\
\text { allele in Ctrl }\end{array}$ & $P$-value & OR $[95 \% \mathrm{Cl}]$ \\
\hline \multirow{3}{*}{$\begin{array}{l}\text { chr2: 106,246, } \\
527-106,251,789\end{array}$} & \multirow{3}{*}{$\begin{array}{l}69 \mathrm{~kb} \text { upstream } \\
\text { of UXS1 }\end{array}$} & discovery $^{a}$ & $861(90.1)$ & $95(9.9)^{*}$ & $6026(79.3)$ & $1570(20.7)^{*}$ & $2.84 \times 10^{-15}$ & $2.36[1.90-2.94]$ \\
\hline & & $\begin{array}{l}\text { unfiltered } \\
\text { discovery }^{b}\end{array}$ & $635(66.4)$ & 321 (33.6) & $5145(67.7)$ & 2451 (32.3) & 0.44 & 0.94 [0.82-1.09] \\
\hline & & replication & 331 (66.5) & $167(33.5)^{*}$ & $594(66.0)$ & $306(34.0)^{*}$ & 0.94 & $1.02[0.81-1.29]$ \\
\hline
\end{tabular}

Deletion is marked by *. The $p$-value corresponds to a ${ }^{2} 2$ test of 478 PsA patients and 3798 control individuals. Odds ratios [95\% confidence interval] of the discovery study (array-based analysis) were calculated for the same cohorts. The independent replication cohorts (MLPA-based analysis) comprised 251 patients and 451 control individuals. ${ }^{2}$ Discovery describes the initial SNP array-based analysis with the filter criteria of 5 markers and $5 \mathrm{~kb}$ before validation, ${ }^{\mathrm{b}}$ unfiltered discovery the number of CNVs and wildtype-alleles in the initial SNP array-based dataset that was unfiltered for no. of markers and for size, as analyzed after validation with MLPA 
Table 2 Allele counts (frequencies) of CNV at TRB in PSA patients and controls (Ctrl)

\begin{tabular}{lllllll}
\hline part of study & $\begin{array}{l}\mathrm{n}(\%) \text {-risk } \\
\text { allele in PSA }\end{array}$ & $\begin{array}{l}\mathrm{n}(\%) \text {-non-risk } \\
\text { allele in PsA }\end{array}$ & $\begin{array}{l}\mathrm{n}(\%) \text {-risk } \\
\text { allele in Ctrl }\end{array}$ & $\begin{array}{l}\mathrm{n}(\%) \text { - non-risk } \\
\text { allele in Ctrl }\end{array}$ & $\begin{array}{l}\text { P-value } \\
\text { OR [95\% CI] }\end{array}$ \\
\hline discovery $^{\mathrm{a}}$ & $776(81.2)$ & $180(18.8)^{*}$ & $5288(69.6)$ & $2308(30.4)^{*}$ & $1.22 \times 10^{-13}$ & $1.88[1.59-2.23]$ \\
extended PSA, independent Ctrl cohort $^{801(51.1)}$ & $765(48.9)^{*}$ & $947(52.8)$ & $847(47.2)^{*}$ & 0.34 & $0.94[0.82-1.07]$ \\
\hline
\end{tabular}

Deletion is marked by *. The $p$-value corresponds to a $x 2$ test of of 478 patients and 3798 control individuals. Odds ratio [ $95 \%$ confidence interval] for the discovery study (array-based analysis) were calculated for the same cohorts. The replication cohort (MLPA-based results) was partially overlapping for PsA, and consisted of 782 patients and 897 controls. ${ }^{a}$ Discovery describes the initial SNP array-based analysis with the filter criteria of 5 markers and 5 kb before validation with MLPA

annotation experimentally, we designed PCR products spanning the region of interest that were specific to either hg19 or hg18 (Fig. 2c, g). Using doubly genotyped PsA patients (i.e. genotyped with both the SNP array and MLPA) as a reference, we also genotyped control individuals by MLPA. Representative carriers of the different copy number states were analysed further by specific amplification and sequencing of PCR products from the TRB region. All three PCR products specific to the hg18 freeze, but not overlapping the deletion, could be amplified in individuals of all three copy number states. By contrast, the product from within the TRB deletion was absent in all homozygous deletion carriers (Fig. 2e, g), as was to be expected. In the hg19 freeze, no explicit sequence ("NNNNNN") was annotated between the sequences that were present in both assemblies in the human genome (represented as black or grey boxes in Fig. 2b). Therefore, we designed PCR products specific to the hg19 freeze spanning these regions (Fig. 2c). However, we could not amplify any specific products in individuals of any copy number state, thereby providing evidence in favour of the hg18 assembly being closer to the actual sequence at this locus than hg19.

The 33 of 48 PsA samples with discordant SNP arraybased and MLPA-based genotypes were reanalysed by PCR specific to the hg18 assembly. We identified 12 deletion homozygous patients through the lack of the PCR product. These genotypes were in accordance with MLPA, but not with the SNP array. Since PCR-based analysis could not distinguish between 1 or 2 copies, we could not evaluate the $\mathrm{CN}$ states of the remaining 23 samples. All of them showed either 1 or 2 copies when genotyped by MLPA. When we re-analysed the complete PsA cohort of the discovery study with MLPA, a similarly high discordance rate emerged between array and MLPA analysis (157 of 446 samples; $35.2 \%$ ) as in the initially analysed subset of patients.

Since genotyping of the CNV appeared to be more reliable with MLPA than array-based data, we screened independent cohorts of 304 patients and 897 healthy controls with MLPA. When comparing the allele frequencies of all patients $(n=782)$ to those in independent

Table 3 Low-frequency CNVs at psoriasis susceptibility loci in PsA patients $(n=478)$ and controls $(n=3798)(C t r l)$

\begin{tabular}{|c|c|c|c|c|c|c|c|}
\hline nearest gene/ locus & type of aberration & $\begin{array}{l}\text { no. of CNV } \\
\text { alleles (PsA) }\end{array}$ & $\begin{array}{l}\text { frequency } \\
\text { in \% (PsA) }\end{array}$ & $\begin{array}{l}\text { validation (no. of PSA } \\
\text { patients analyzed by MLPA) }\end{array}$ & $\begin{array}{l}\text { no. of CNV } \\
\text { alleles (Ctrl) }\end{array}$ & $\begin{array}{l}\text { frequency } \\
\text { in \% (Ctrl) }\end{array}$ & $p$-value \\
\hline CSMD1 & deletion & 1 & 0.10 & validated (7) & 7 & 0.09 & 1 \\
\hline $\operatorname{IL12B}$ & duplication & 2 & 0.21 & validated (14) & 3 & 0.04 & 0.1 \\
\hline RYR2 & deletion & 2 & 0.21 & validated (7) & 12 & 0.16 & 0.7 \\
\hline ERAP1 & deletion & 2 & 0.21 & not validated (7) & 1 & 0.01 & $\mathrm{n} / \mathrm{a}$ \\
\hline ERAP1 & duplication & 1 & 0.10 & not validated (7) & 4 & 0.05 & $\mathrm{n} / \mathrm{a}$ \\
\hline FBXL19 & deletion & 1 & 0.10 & not validated (14) & 0 & 0 & $\mathrm{n} / \mathrm{a}$ \\
\hline FBXL19 & duplication & 2 & 0.21 & not validated (14) & 22 & 0.33 & $\mathrm{n} / \mathrm{a}$ \\
\hline GJB2 & duplication & 1 & 0.10 & not validated (14) & 1 & 0.01 & $\mathrm{n} / \mathrm{a}$ \\
\hline$|F| H 1$ & duplication & 3 & 0.31 & not validated (14) & 6 & 0.09 & $\mathrm{n} / \mathrm{a}$ \\
\hline IL23A & duplication & 4 & 0.42 & not validated (7) & 8 & 0.12 & $\mathrm{n} / \mathrm{a}$ \\
\hline NFKBIA & duplication & 2 & 0.21 & not validated (14) & 6 & 0.09 & $\mathrm{n} / \mathrm{a}$ \\
\hline RNF114 & duplication & 2 & 0.31 & not validated (12) & 7 & 0.09 & $\mathrm{n} / \mathrm{a}$ \\
\hline TNIP1 & duplication & 1 & 0.10 & not validated (7) & 0 & 0 & $n / a$ \\
\hline TRAF3IP2 & duplication & 3 & 0.31 & not validated (12) & 7 & 0.09 & $\mathrm{n} / \mathrm{a}$ \\
\hline
\end{tabular}

Gene nearest to the susceptibility locus, the type of aberration, the absolute no. of CNV alleles, their overall frequency in percent, the result of a validation by MLPA (in PsA patients only) and the results of a Fisher's exact test (n/a: not applicable due to lack of validation) are given 


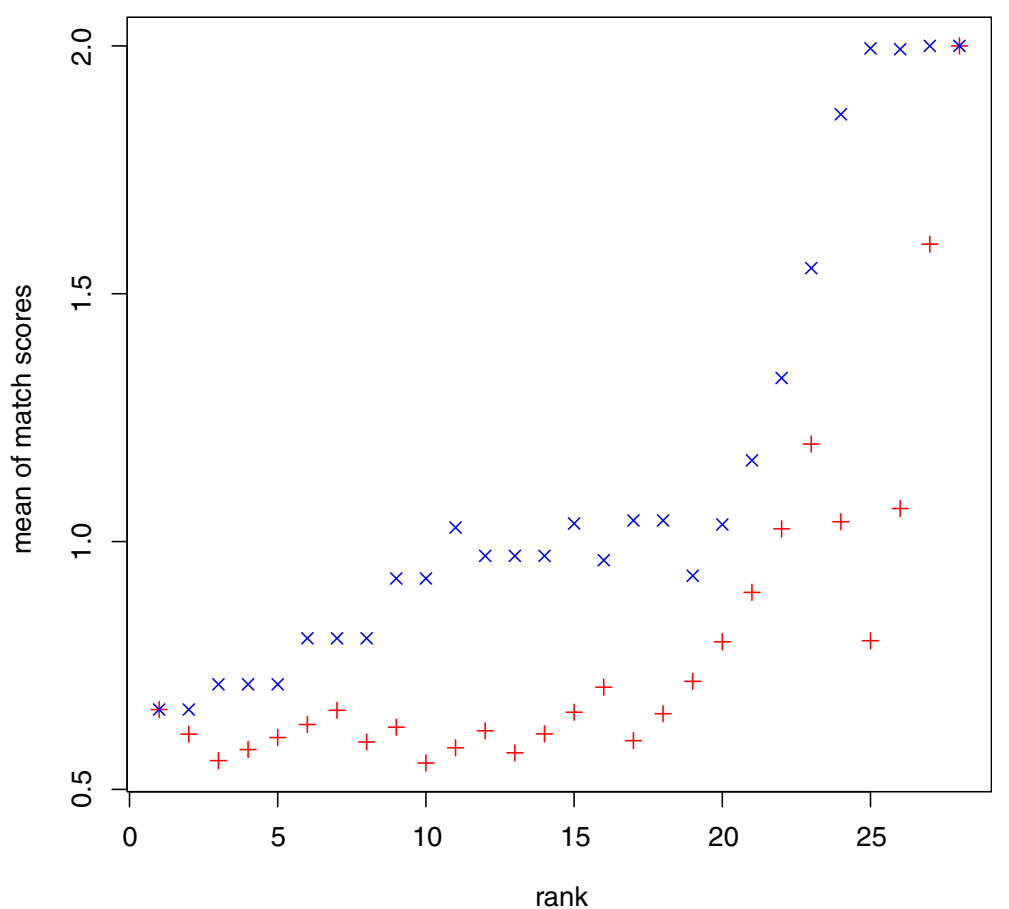

Fig. 3 Correlation between degree of copy number validation and median size and marker count of CNVs. X axis: ranking of 28 CNVs (8 frequent, 20 low-frequency) according to median size (red vertical crosses) or marker count (blue diagonal crosses); $Y$ axis: mean of mean match scores for this CNV and all CNVs with a higher size or marker count

control individuals $(n=897)$, no significant difference was observed (Table 2). Frequencies observed in the MLPA-based second analysis were significantly different from those of the discovery study (Table 2), suggesting difficulties in genotyping this CNV by arraybased analyses.

\section{CNVs at psoriasis susceptibility loci}

Of note, we did not detect association to the CNVs at previously identified copy number loci such as $L C E 3 C /$ $L C E 3 B$ [5], the $\beta$-defensin-cluster $[19,20]$ and the intergenic deletion [25]. As we had not observed association to the $L C E 3 C / L C E 3 B$ in a largely overlapping cohort of 650 German PsA patients [34], we did not expect to find evidence for association. Furthermore, the SNP array-based analysis could not adequately and reliably disentangle the multivariable $ß$-defensin cluster. Last but not least, the minor allele frequency of the intergenic CNV was similar in cases (8\%) and controls (7.5\%). More detailed results on the $L C E 3$ cluster and the $\beta$-defensin cluster can be read elsewhere [27].

By using the SNP array-based approach to detect frequent $\mathrm{CNVs}$ at 30 previously identified susceptibility loci for PsV or PsA [4-11, 13, 16], we did not detect any. Therefore, CNVs at these loci do not seem to be reasonable candidate variants for disease-causality, at least not in German patients with PsA, but we have to consider the limitations of our method with regard to smaller sizes of $(<5000 \mathrm{bp})$ and the detection rate of $\mathrm{CNVs}$ using the procedure of SNP array-based analyses. One frequent deletion was identified at the $H L A-C$ locus, but owing the high level of sequence homology (> 99\%) to the HLA-B locus, we were unable to technically validate this CNV by MLPA. Therefore, it remained unclear whether the disease association of this locus was genuine or represented a technical artefact.

We exclusively identified low-frequency CNVs $(\mathrm{MAF}<5 \%)$ at 12 of the 30 psoriasis susceptibility loci studied, with duplications $(n=16)$ being more frequent than deletions $(n=4)$ (Table 3$)$. Only four different CNVs were confirmed by MLPA, namely two intronic deletions in the RYR2and CSMD1 genes and two duplications at the $I L 12 B$ locus. In case of CNVs at the remaining susceptibility loci, no copy number variability could be confirmed. We also observed lowfrequency CNVs at the three loci in control individuals, but no evidence for disease association was apparent (Table 3).

\section{CNV characteristics and MLPA validation}

We performed MLPA for eight frequent CNVs identified through GWAS (see Tables 1 and 2; [27]) and for 20 low-frequency CNVs at psoriasis susceptibility loci (Table 3; Additional file 1: Table S2) and compared the 
outcome to CNV calling from SNP array data (frequent CNVs [27]; low-frequency CNVs: Table 3; Additional file 1: Table S2). A significant correlation was observed between the type of aberration (deletion versus duplication) and the chance of MLPA validation $\left(p=3.01 \times 10^{-3}\right)$. Deletions were validated more often ( 8 of $11,73 \%$ ) than duplications ( 2 of $17,12 \%)$, as is also reflected by the difference in the mean of mean match scores (1.32 for deletions; 0.24 for duplications).

We also observed evidence for a positive correlation between the mean match score of CNVs and its median segment size (Spearman's $\rho=0.77$ ) and an almost perfect correlation between the mean match score and the median marker count (Spearman's $\rho=0.96$; Fig. 2). Although larger $\mathrm{CNVs}$ comprising higher marker counts showed a higher level of concordance between SNP array-based genotype and MLPA-based genotype, a lower marker count or a smaller size of a CNV did not indicate discordance between SNP array-based and MLPA-based genotypes in case of all small CNVs.

\section{Discussion}

Although CNVs have been shown to potentially play a role in the genetic basis of both PsV and PsA [5, 19, 20, 22, 25], our GWAS of CNVs failed to identify any novel disease-associated $\mathrm{CNV}$ for PsA. As a result of our three-stage analysis comprising thorough quality assessment [27], validation by MLPA as a second quantitative method and replication analysis in an enlarged casecontrol study, none of the originally identified diseaseassociations of CNVs remained significant. In view of previous experience with small CNVs in the context of immunological diseases (e.g. type 2 diabetes, Crohn's disease and rheumatoid arthritis [35]), we chose to screen CNVs of 5000 bp or more. This notwithstanding, even if we considered small CNVs, no robust association between PsA and a frequent $\mathrm{CNV}$ could be found.

Previous studies reported on lower recovery rates and pronounced difficulties in genotyping frequent CNVs $[36,37]$, especially in the case of full gene deletions. The same reasons responsible in these instances may also explain the discrepancies in CNV genotyping between SNP array and MLPA noted in our study for the frequent deletion at the TRB locus.

Our analysis revealed differences in annotation of the $T R B$ locus on chromosome $7 \mathrm{q} 34$ between genome freezes hg18 and hg19, and experimental evidence was generated in favour of hg18 being closer to the true DNA sequence. Through intensive investigation of this locus, we could validate a frequent $\mathrm{CNV}$ at TRB, but its initial association with PsA, probably due to wrong genotypes derived for the SNP array-based analysis could not be replicated. The locus on chromosome $7 \mathrm{q} 34$ is complex and comprises numerous annotated UCSC genes and transcript variants. T cell receptors are known to undergo somatic rearrangements of variable $(\mathrm{V})$, diverse $(\mathrm{D})$ and joining $(\mathrm{J})$ gene segment/ chains $(=\mathrm{V}(\mathrm{D}) \mathrm{J}$ recombination). Moreover, an enormous variability in terms of the clonality of T-cell populations exists between individuals and increases the complexity of this genomic locus [38, 39]. In summary, high variability and lack of representativeness of the reference sequences might have contributed notably to the difficulties in SNP array-based genotyping of the TRB cluster.

We failed to identify association of PsA with a novel CNV. This might be due to the limited power that we had in 478 patients, while we cannot exclude that we missed associations to CNVs due to the limitations of the SNP array-based analysis.

Our study strongly suggests that independent validation of CNVs is essential for their use as disease markers, particularly because the low-frequency CNVs considered at psoriasis susceptibility loci in our study were characterized by an unacceptably low level of validation. Low-frequency duplications were remarkably more difficult to validate than low-frequency deletions. Moreover, because no frequent duplication was validated at all, we assume that the detection of duplications is generally more complicated and less reliable than that of deletions. These findings are in accordance with previous findings, for example of Zhang et al. [36] who also reported lower recovery rates for duplications. In addition, recovery rates for computer-simulated duplications were found to be lower than those for equally sized deletions [40].

We observed a robust correlation between both the number of markers involved and the size of CNVs on the one hand and the level at which these CNVs are successfully validated. The impact of marker count and CNV size on the reliability of CNV calling has been discussed before $[36,39,41]$ and was quantified by simulation [40], indicating that recovery rates of CNVs increase when the marker count increases. This increase was also shown to be more rapid for deletions than for duplications.

\section{Conclusions}

Although we were able to detect an initial PsA association for two CNVs and could confirm that the frequent CNVs involved were real, we failed to validate the copy number states in a significant number of cases. Also, we were unable to replicate the original disease association. Furthermore, SNP array-based analyses of CNVs are more reliable in pinpointing deletions than duplications, independent of the $\mathrm{CNV}$ allele frequency. Frequent CNVs remain good candidate variants for disease association, while we suggest to use also other methods to detect CNVs cautiously and to reproduce CNVs with an 
independent method. Other than SNP array-based analyses, e.g. digital PCR, might be more useful to detect CNVs.

\section{Additional file}

Additional file 1: Tables $\mathbf{S 1}$ and $\mathbf{S 2}$ are presented in the Supplementary data file. (DOCX $25 \mathrm{~kb}$ )

\section{Abbreviations}

CNVs: Copy number variants; Ctrl: Control individuals; DEFB: Beta-defensins; del: Deletion; dup: Duplication; GWAS: Genome-wide association study/ ies; LCE3: Late cornified envelope group 3; LD: Linkage disequilibrium; MLPA: Multiplex ligation-dependent probe amplification; no.: Number; PsA: Psoriatic arthritis; PsV: Psoriasis vulgaris; SNPs: Single nucleotide polymorphisms; TRB: T cell receptor beta locus; WT: Wild-type

\section{Acknowledgements}

We are grateful to all patients and control individuals for participation in our study. We thank Petra Badorf for excellent technical assistance and Franziska Degenhardt for helpful discussions.

\section{Funding}

This work was supported in part by a grant from the ELAN fund (Erlanger Leistungsbezogene Anschubfinanzierung und Nachwuchsförderung, to U.H.) of the Friedrich-Alexander-Universität Erlangen-Nürnberg, Germany; and by grants from the Interdisciplinary Centre for Clinical Research ( $\mathrm{J} 1$ and laboratory rotation; to U.H.) of the Clinical Center Erlangen of the Friedrich-AlexanderUniversität Erlangen-Nürnberg, Germany, as well as the German Federal Ministry of Education and Research [Bundesministerium für Bildung und Forschung (BMBF); project ANCYLOSS; to A.R. and ArthroMark (project 4, 01EC1401C); to H.B.]. H.B. received funding from Pfizer Pharma, Germany (Forschungsförderpreis Rheumatologie 2012) and F.B. from the Regional Rhein-Main Rheumatology Centre. The SHIP study was supported by grants from the German Federal Ministry of Education and Research [Bundesministerium für Bildung und Forschung (BMBF)]; project 01ZZ9603, $01 Z Z 0103$ and $01 Z Z 0701$ and the Ministry of Cultural Affairs as well as the Social Ministry of the Federal State of Mecklenburg-West Pomerania. Generation of genome-wide data was supported by the BMBF (grant no. 03ZIK012) and a joint grant from Siemens Healthcare (Erlangen, Germany) and the Federal State of MecklenburgWest Pomerania. The University of Greifswald is a member of the 'Center of Knowledge Interchange' program of the Siemens AG and the Caché Campus program of the InterSystems $\mathrm{GmbH}$.

\section{Availability of data and materials}

Data used in this study cannot be made available, as for data protection reasons, SHIP is not allowed to publish participant-related original data. For quality assurance data can be accessed via https://www.fvcm.med.uni-greifswald.de/cm_antrag/index.php

\section{Authors' contributions}

$A R$ and $U H$ designed the study. ME, $A B E, F B, B B, G H, C S, U V, M J, M N, H V, H T$, $\mathrm{HB}, \mathrm{UH}$ contributed to acquisition of data. $\mathrm{FB}, \mathrm{BB}, \mathrm{HB}, \mathrm{AR}$ and $\mathrm{UH}$ were responsible for the cohort of PSA patients, GH, CS, UV, MJ, MN and HV for the SHIP cohort, HT, AR and UH for the independent control cohort. SU, ME, $A B E, G H, C S$, and $U H$ analyzed data, data were interpreted by $S U, M E, A B E$, $M K, A R$ and $U H$. UH wrote the first draft of the manuscript that was revised critically by all authors and approved in its final version.

\section{Ethics approval and consent to participate}

The studies were approved by the ethics committee of medical faculty of the Friedrich-Alexander-Universität Erlangen-Nürnberg (FAU) (Re.-No. 4597), by the ethics committee of the medical faculty of the University of Münster (Reg.-No. 14.07.95/ 21.07.03), by the ethics committee of the Medical Faculty, of the Goethe University in Frankfurt am Main (Re.-No. 283/07) and by the ethics committee of the medical faculty of the Ernst-Moritz-Arndt-Universität Greifswald (Re.-No. BB 39/08 and III UV 37/03b). All participants gave written informed consent. Investigations were conducted according to the Declaration of Helsinki principles.

\section{Consent for publication}

Not applicable for this study.

\section{Competing interests}

The authors declare that there are no financial and no non-financial competing interests.

\section{Publisher's Note}

Springer Nature remains neutral with regard to jurisdictional claims in published maps and institutional affiliations.

\section{Author details}

${ }^{1}$ Institute of Human Genetics, Friedrich-Alexander-Universität

Erlangen-Nürnberg (FAU), Schwabachanlage 10, 91054 Erlangen, Germany.

${ }^{2}$ Division of Rheumatology and IME Fraunhofer Project Group Translational

Medicine \& Pharmacology, Goethe University, Frankfurt/Main, Germany,

${ }^{3}$ Interfaculty Institute for Genetics and Functional Genomics, University

Medicine and Ernst-Moritz-Arndt University Greifswald, Greifswald, Germany.

${ }^{4}$ Clinic of Dermatology, University of Greifswald, Greifswald, Germany.

${ }^{5}$ Institute of Clinical Chemistry and Laboratory Medicine, University of Greifswald, Greifswald, Germany. ${ }^{6}$ Institute for Community Medicine, University of Greifswald, Greifswald, Germany. ${ }^{7}$ Department of Dermatology, University of Münster, Münster, Germany. ${ }^{8}$ Institute for Medical Informatics and Statistics, Christian-Albrechts University Kiel, Kiel, Germany.

Received: 4 March 2016 Accepted: 31 July 2017

Published online: 23 August 2017

\section{References}

1. Chandran V, Schentag CT, Brockbank JE, Pellett FJ, Shanmugarajah S, Toloza SM, Rahman P, Gladman DD. Familial aggregation of psoriatic arthritis. Ann Rheum Dis. 2009;68(5):664-7.

2. Karason A, Love TJ, Gudbjornsson B. A strong heritability of psoriatic arthritis over four generations-the Reykjavik psoriatic arthritis study. Rheumatology (Oxford). 2009;48(11):1424-8.

3. Di Lernia V, Ficarelli E, Lallas A, Ricci C. Familial aggregation of moderate to severe plaque psoriasis. Clin Exp Dermatol. 2014;

4. Capon F, Bijlmakers MJ, Wolf N, Quaranta M, Huffmeier U, Allen M, Timms K, Abkevich V, Gutin A, Smith R, et al. Identification of ZNF313/RNF114 as a novel psoriasis susceptibility gene. Hum Mol Genet. 2008;17(13):1938-45.

5. de Cid R, Riveira-Munoz E, Zeeuwen PL, Robarge J, Liao W, Dannhauser EN, Giardina E, Stuart PE, Nair R, Helms C, et al. Deletion of the late cornified envelope LCE3B and LCE3C genes as a susceptibility factor for psoriasis. Nat Genet. 2009;41(2):211-5.

6. Ellinghaus E, Ellinghaus D, Stuart PE, Nair RP, Debrus S, Raelson JV, Belouchi M, Fournier H, Reinhard C, Ding J, et al. Genome-wide association study identifies a psoriasis susceptibility locus at TRAF3IP2. Nat Genet. 2010;42(11):991-5.

7. Nair RP, Duffin KC, Helms C, Ding J, Stuart PE, Goldgar D, Gudjonsson JE, Li Y, Tejasvi T, Feng BJ, et al. Genome-wide scan reveals association of psoriasis with IL-23 and NF-kappaB pathways. Nat Genet. 2009;41(2):199-204.

8. Strange A, Capon F, Spencer CC, Knight J, Weale ME, Allen MH, Barton A, Band G, Bellenguez C, Bergboer JG, et al. A genome-wide association study identifies new psoriasis susceptibility loci and an interaction between HLA-C and ERAP1. Nat Genet. 2010:42(11):985-90.

9. Stuart PE, Nair RP, Ellinghaus E, Ding J, Tejasvi T, Gudjonsson JE, Li Y, Weidinger S, Eberlein B, Gieger C, et al. Genome-wide association analysis identifies three psoriasis susceptibility loci. Nat Genet. 2010;42(11):1000-4.

10. Tsoi LC, Spain SL, Knight J, Ellinghaus E, Stuart PE, Capon F, Ding J, Li Y, Tejasvi T, Gudjonsson JE, et al. Identification of 15 new psoriasis susceptibility loci highlights the role of innate immunity. Nat Genet. 2012;44(12):1341-8.

11. Sun LD, Cheng H, Wang ZX, Zhang AP, Wang PG, Xu JH, Zhu QX, Zhou HS, Ellinghaus $E$, Zhang $F R$, et al. Association analyses identify six new psoriasis susceptibility loci in the Chinese population. Nat Genet. 2010;42(11):1005-9.

12. Zhang XJ, Huang W, Yang S, Sun LD, Zhang FY, Zhu QX, Zhang FR, Zhang C, Du WH, Pu XM, et al. Psoriasis genome-wide association study identifies susceptibility variants within LCE gene cluster at 1q21. Nat Genet. 2009;41(2):205-10.

13. Apel M, Uebe S, Bowes J, Giardina E, Korendowych E, Juneblad K, Pasutto F, Ekici $A B$, McManus R, Ho $P$, et al. Variants in RUNX3 contribute to susceptibility to psoriatic arthritis, exhibiting further common ground with Ankylosing Spondylitis. Arthritis Rheum. 2013;65(5):1224-31. 
14. Bowes J, Budu-Aggrey A, Huffmeier U, Uebe S, Steel K, Hebert HL, Wallace C, Massey J, Bruce IN, Bluett J, et al. Dense genotyping of immune-related susceptibility loci reveals new insights into the genetics of psoriatic arthritis. Nat Commun. 2015;6:6046.

15. Bowes J, Loehr S, Budu-Aggrey A, Uebe S, Bruce IN, Feletar M, Marzo-Ortega H, Helliwell P, Ryan AW, Kane D, et al. PTPN22 is associated with susceptibility to psoriatic arthritis but not psoriasis: evidence for a further PsA-specific risk locus. Ann Rheum Dis. 2015;74:1882-5.

16. Huffmeier U, Uebe S, Ekici AB, Bowes J, Giardina E, Korendowych E, Juneblad K, Apel M, McManus R, Ho P, et al. Common variants at TRAF3IP2 are associated with susceptibility to psoriatic arthritis and psoriasis. Nat Genet. 2010;42(11):996-9.

17. lafrate AJ, Feuk L, Rivera MN, Listewnik ML, Donahoe PK, Qi Y, Scherer SW, Lee $\mathrm{C}$. Detection of large-scale variation in the human genome. Nat Genet. 2004;36(9):949-51.

18. Sebat J, Lakshmi B, Troge J, Alexander J, Young J, Lundin P, Maner S, Massa $\mathrm{H}$, Walker M, Chi M, et al. Large-scale copy number polymorphism in the human genome. Science. 2004;305(5683):525-8.

19. Hollox EJ, Huffmeier U, Zeeuwen PL, Palla R, Lascorz J, Rodijk-Olthuis D, van de Kerkhof PC, Traupe H, de Jongh G, den Heijer M, et al. Psoriasis is associated with increased beta-defensin genomic copy number. Nat Genet. 2008;40(1):23-5.

20. Stuart PE, Huffmeier U, Nair RP, Palla R, Tejasvi T, Schalkwijk J, Elder JT, Reis A, Armour JA. Association of beta-defensin copy number and psoriasis in three cohorts of European origin. The Journal of investigative dermatology. 2012;132(10):2407-13.

21. Huffmeier U, Bergboer JG, Becker T, Armour JA, Traupe H, Estivill X, RiveiraMunoz E, Mossner R, Reich K, Kurrat W, et al. Replication of LCE3C-LCE3B $C N V$ as a risk factor for psoriasis and analysis of interaction with other genetic risk factors. The Journal of investigative dermatology. 2010;130(4): 979-84.

22. Docampo E, Giardina E, Riveira-Munoz E, de Cid R, Escaramis G, Perricone C, Fernandez-Sueiro JL, Maymo J, Gonzalez-Gay MA, Blanco FJ, et al. Deletion of LCE3C and LCE3B is a susceptibility factor for psoriatic arthritis: a study in Spanish and Italian populations and meta-analysis. Arthritis Rheum. 2011;63(7):1860-5.

23. Riveira-Munoz E, He SM, Escaramis G, Stuart PE, Huffmeier U, Lee C, Kirby B, Oka A, Giardina E, Liao W, et al. Meta-analysis confirms the LCE3C_LCE3B deletion as a risk factor for psoriasis in several ethnic groups and finds interaction with HLA-Cw6. The Journal of investigative dermatology. 2011; 131(5):1105-9.

24. Bowes J, Flynn E, Ho P, Aly B, Morgan AW, Marzo-Ortega H, Coates L, McManus R, Ryan AW, Kane D, et al. Variants in linkage disequilibrium with the late cornified envelope gene cluster deletion are associated with susceptibility to psoriatic arthritis. Ann Rheum Dis. 2010;69(12):2199-203.

25. Julia A, Pinto JA, Gratacos J, Queiro R, Ferrandiz C, Fonseca E, Montilla C, Torre-Alonso JC, Puig L, Perez Venegas JJ, et al. A deletion at ADAMTS9MAGI1 locus is associated with psoriatic arthritis risk. Ann Rheum Dis. 2015;74(10):1875-81.

26. Volzke H, Alte D, Schmidt CO, Radke D, Lorbeer R, Friedrich N, Aumann N, Lau K, Piontek M, Born G, et al. Cohort profile: the study of health in Pomerania. Int J Epidemiol. 2011;40(2):294-307.

27. Ehrlicher M, Uebe S, Krumbiegel M, Ekici AB, Homuth G, Schurmann C, Völker U, Jünger M, Nauck M, Völzke H et al: Quality assessment of diseaseassociated CNVs derived from SNP array-based GWAS data. 2017, submitted.

28. Price AL, Patterson NJ, Plenge RM, Weinblatt ME, Shadick NA, Reich D. Principal components analysis corrects for stratification in genome-wide association studies. Nat Genet. 2006;38(8):904-9.

29. Huffmeier U, Lascorz J, Becker T, Schurmeier-Horst F, Magener A, Ekici AB, Endele S, Thiel CT, Thoma-Uszynski S, Mossner R, et al. Characterisation of psoriasis susceptibility locus 6 (PSORS6) in patients with early onset psoriasis and evidence for interaction with PSORS1. J Med Genet. 2009;46(11):736-44.

30. Huffmeier U, Lascorz J, Bohm B, Lohmann J, Wendler J, Mossner R, Reich K, Traupe $H$, Kurrat W, Burkhardt $H$, et al. Genetic variants of the IL-23R pathway: association with psoriatic arthritis and psoriasis vulgaris, but no specific risk factor for arthritis. J Invest Dermatol. 2009;129(2):355-8.

31. Korn JM, Kuruvilla FG, McCarroll SA, Wysoker A, Nemesh J, Cawley S, Hubbell E, Veitch J, Collins PJ, Darvishi K, et al. Integrated genotype calling and association analysis of SNPS, common copy number polymorphisms and rare CNVs. Nat Genet. 2008;40(10):1253-60.

32. Korber A, Mossner R, Renner R, Sticht H, Wilsmann-Theis D, Schulz P, Sticherling $M$, Traupe $H$, Huffmeier U. Mutations in IL36RN in patients with generalized pustular psoriasis. J Invest Dermatol. 2013;133(11):2634-7.
33. Gabriel SB, Schaffner SF, Nguyen H, Moore JM, Roy J, Blumenstiel B, Higgins J, DeFelice M, Lochner A, Faggart M, et al. The structure of haplotype blocks in the human genome. Science. 2002;296(5576):2225-9.

34. Huffmeier U, Estivill X, Riveira-Munoz E, Traupe H, Wendler J, Lohmann J, Bohm B, Burkhardt H, Reis A. Deletion of LCE3C and LCE3B genes at PSORS4 does not contribute to susceptibility to psoriatic arthritis in German patients. Ann Rheum Dis. 2010;69(5):876-8.

35. Craddock N, Hurles ME, Cardin N, Pearson RD, Plagnol V, Robson S, Vukcevic D, Barnes C, Conrad DF, Giannoulatou E, et al. Genome-wide association study of CNVs in 16,000 cases of eight common diseases and 3,000 shared controls. Nature. 2010;464(7289):713-20.

36. Zhang D, Qian Y, Akula N, Alliey-Rodriguez N, Tang J, Bipolar Genome S, Gershon ES, Liu C. Accuracy of CNV detection from GWAS data. PLoS One. 2011;6(1):e14511.

37. Marenne G, Real FX, Rothman N, Rodriguez-Santiago B, Perez-Jurado L, Kogevinas M, Garcia-Closas M, Silverman DT, Chanock SJ, Genin E, et al. Genome-wide CNV analysis replicates the association between GSTM1 deletion and bladder cancer: a support for using continuous measurement from SNP-array data. BMC Genomics. 2012;13:326.

38. Groenen PJ, Langerak AW, van Dongen JJ, van Krieken JH. Pitfalls in TCR gene clonality testing: teaching cases. J Hematop. 2008;1(2):97-109.

39. Glessner JT, Li J, Hakonarson H. ParseCNV integrative copy number variation association software with quality tracking. Nucleic Acids Res. 2013;41(5):e64.

40. Wineinger NE, Tiwari HK. The impact of errors in copy number variation detection algorithms on association results. PLoS One. 2012;7(4):e32396.

41. Asadollahi R, Oneda B, Joset $P$, Azzarello-Burri S, Bartholdi D, Steindl K, Vincent M, Cobilanschi J, Sticht H, Baldinger R, et al. The clinical significance of small copy number variants in neurodevelopmental disorders. J Med Genet. 2014;51(10):677-88.

\section{Submit your next manuscript to BioMed Central and we will help you at every step:}

- We accept pre-submission inquiries

- Our selector tool helps you to find the most relevant journal

- We provide round the clock customer support

- Convenient online submission

- Thorough peer review

- Inclusion in PubMed and all major indexing services

- Maximum visibility for your research

Submit your manuscript at www.biomedcentral.com/submit
C Biomed Central 